\title{
$B R A F$ mutation is associated with DNA methylation in serrated polyps and cancers of the colorectum
}

\author{
T Kambara, L A Simms, V L J Whitehall, K J Spring, C V A Wynter, M D Walsh, M A Barker, \\ S Arnold, A McGivern, N Matsubara, N Tanaka, T Higuchi, J Young, J R Jass, B A Leggett
}

Gut 2004;53:1137-1144. doi: 10.1136/gut.2003.037671

See end of article for authors' affiliations

Correspondence to: Dr B A Leggett, Conjoint Gastroenterology Laboratory, Royal Brisbane and Women's Hospital Research Foundation and Queensland Institute of Medical Research, Brisbane 4029, Australia; Barbara_Leggett@ health.qld.gov.au

Revised version received 3 February 2004 Accepted for publication 17 February 2004

\begin{abstract}
Background and aims: Mutations in BRAF have been linked with colorectal cancers (CRC) showing high level microsatellite instability (MSI-H). However, the distribution of BRAF mutations in MSI-H cancers remains to be clarified with respect to precursor lesions and the CpG island methylator phenotype (CIMP). Methods: Forty three hyperplastic polyps (HP), nine mixed polyps (MP), five serrated adenomas (SA), 28 conventional adenomas (AD), 18 hereditary non-polyposis colorectal cancers (HNPCC), and 127 sporadic CRC (46 MSI-H and 81 non-MSI-H) were collected from patients undergoing colectomy for either CRC or hyperplastic polyposis. Twenty five of 57 serrated lesions were derived from four patients with hyperplastic polyposis. HP were further subdivided according to recently documented morphological criteria into 27 classical HP and 16 variant lesions described as "sessile serrated adenoma" (SSA). All tumours were screened for $B R A F$ activating mutations.

Results: The BRAF mutation was more frequent in SSA $(75 \%)$ and MP (89\%) than in classical HP (19\%), SA $(20 \%)$, and $A D(0 \%)(p<0.0001)$, and also in sporadic MSI-H cancers $(76 \%)$ compared with HNPCC $(0 \%)$ and sporadic non-MSI-H cancers (9\%) ( $\mathrm{p}<0.0001)$. The BRAF mutation was identified more offen in CIMPhigh serrated polyps (72\%) and CIMP-high CRC (77\%) than in CIMP-low (30\%) and CIMP-negative (13\%) polyps $(p=0.002)$ as well as CIMP-low $(18 \%)$ and CIMP-negative $(0 \%)$ CRC $(p<0.0001)$.

Conclusions: The BRAF mutation was frequently seen in SSA and in sporadic MSI-H CRC, both of which were associated with DNA methylation. Sporadic MSI-H cancers may originate in SSA and not adenomas, and BRAF mutation and DNA methylation are early events in this "serrated" pathway.
\end{abstract}

\footnotetext{
C
} olorectal cancer (CRC) is one of the leading causes of cancer mortality in the Western world and is considered to be a heterogeneous disease..$^{1-3}$ Approximately $5 \%$ of cases are attributable to familial cancer syndromes (hereditary non-polyposis colorectal cancers (HNPCC) and familial adenomatous polyposis (FAP)) while the remainder arises sporadically. ${ }^{12}$ HNPCC is due to a germline mutation of DNA mismatch repair (MMR) genes, and cancers occurring in this condition show high level DNA microsatellite instability (MSI-H). Approximately $15 \%$ of sporadic CRC are also characterised by high level MSI due to somatic inactivation of a MMR gene. ${ }^{2}$ While the evolution of the majority of non-MSI-H cancers is consistent with the accepted adenoma-carcinoma model, ${ }^{4}$ initiation and pathogenic progression of sporadic MSI-H cancers remains controversial. $^{356}$

For many years, hyperplastic polyps (HP) have been regarded as colorectal lesions with little neoplastic potential and therefore of no pathogenic consequence. ${ }^{7}$ However, this view has recently been challenged by the proposal that a subset of HP predisposes to sporadic MSI-H cancers and may progress through a "serrated neoplastic pathway". ${ }^{3}$ Lesions with serrated architecture (serrated polyps) include classical HP and the much rarer mixed polyps (MP), and serrated adenomas (SA). ${ }^{79}$ SA are composed of adenomatous (dysplastic) epithelium that nevertheless show the architectural serration that is characteristic of HP while MP are combined lesions that include separate hyperplastic and adenomatous components. $^{10}$ Based on multiple microscopic features, classical or harmless HP have recently been distinguished from large, often right sided, HP which are more likely to have malignant potential. ${ }^{91-13}$

An important molecular feature frequently associated with, but not exclusive to, sporadic MSI-H cancers is the CpG island methylator phenotype (CIMP) which is characterised by methylation of multiple $\mathrm{CpG}$ islands located within the promoter region of genes. ${ }^{14-18} \mathrm{CpG}$ islands are seen in approximately $50 \%$ of human genes and consist of dense regions of cytosine-guanosine dinucleotides that are susceptible to methylation of the cytosine residues resulting in gene silencing. Within the context of CRC, hypermethylation of CpG islands provides an epigenetic mechanism for the transcriptional repression of genes which are known to be important in neoplastic development, such as pl6, MGMT, and the MMR gene $h M L H 1 .{ }^{14}{ }^{19-21}$ It has been postulated that aberrant methylation in association with other genetic alterations is the mechanism by which the "serrated neoplastic pathway" to CRC is initiated. ${ }^{322}$

BRAF, a member of the RAF family of serine/threonine kinases, mediates cellular responses to growth signals through the RAS-RAF-MAP kinase pathway. ${ }^{23}{ }^{24}$ Activating mutations in BRAF have recently been identified in $70 \%$ of malignant melanomas and $5-15 \%$ of $\mathrm{CRCs}^{25-27}$ In all of the human cancers surveyed, the most common mutation identified was a V599E amino acid substitution that mimics phosphorylation at T598 and S601 and results in constitutive kinase activation..$^{25}$ Furthermore, it has been demonstrated that the BRAF mutation is strongly associated with MMR deficient CRC and inversely correlated with mutation of the $\mathrm{K}$-ras oncogene. ${ }^{25-27}$ Based on this evidence it was postulated

Abbreviations: $A D$, conventional adenoma (tubular adenoma and tubulovillous adenoma); CIMP, CpG island methylator phenotype; CRC, colorectal cancer; $\mathrm{AHPLC}$, denaturing high performance liquid chromatography; FAP, familial adenomatous polyposis; HNPCC, hereditary non-polyposis colorectal cancer; HP, hyperplastic polyp MMR, mismatch repair; MP, mixed polyp; MSI, microsatellite instability; $\mathrm{MSI}-\mathrm{H}$, high level MSI; RFLP, restriction fragment length polymorphism; $\mathrm{SA}$, serrated adenoma; SSA, sessile serrated adenoma 
that BRAF and K-ras mutations are equivalent in their tumorigenic effects and that both MMR deficient and proficient tumour types progress through the same biochemical pathways. ${ }^{26}$

In this study, we evaluated the frequency of the BRAF mutation in 85 colorectal polyps, and in 145 CRC stratified by MSI and/or CIMP status. Our findings showed that the oncogenic BRAF mutation had a striking association with both CIMP-high sporadic MSI-H cancers and a subset of HP showing CIMP-high, thereby providing strong evidence that sporadic MSI-H cancers evolve from these lesions and not from adenomas. These results suggest that the BRAF mutation and DNA methylation are key genetic events in initiation of the serrated neoplastic pathway in the development of CRC.

\section{MATERIALS AND METHODS \\ Tissue samples}

The study was performed on fresh frozen tissue samples obtained from patients undergoing colectomy for neoplasia at the Royal Brisbane Hospital. Patients provided written informed consent and the study was approved by the Human Research Ethics Committee of the Queensland Institute of Medical Research. All samples were dissected macroscopically by a pathologist to ensure that they contained predominantly neoplastic tissue. The study group consisted of 85 colorectal polyps and 145 cancers, both with matching normal mucosa. Age at operation and sex of the patient were noted. Cases were selected for study from a large consecutive series of operative specimens on the basis of MSI status or evidence of HNPCC (cancers) and overall histological appearance (polyps).

The colorectal cancer group was enriched for MSI-H cancers as BRAF activating mutations had been specifically associated with this tumour type, ${ }^{26}$ and included $64 \mathrm{MSI}-\mathrm{H}$ cancers (46 sporadic and 18 HNPCC). Sporadic MSI-H cancers comprised cancers derived from patients over 56 years of age with no family history of CRC. HNPCC tumours had been characterised in a prior study. ${ }^{29}$ MSI status for each tumour was determined by examining a panel of 10 microsatellite markers (BAT-26, BAT-25, BAT-40, BAT-34C4, MYCL, D10S197, D18S55, D5S346, D17S250, and ACTC) using previously described techniques. ${ }^{30-33}$ Cancers with instability in at least four $(40 \%)$ of the markers were classified as MSI-H with the remainder classified as
non-MSI-H. ${ }^{31}$ For all MSI-H cancers, immunohistochemical staining of hMLHl and hMSH2 was performed and all sporadic MSI-H cancers showed loss of hMLHl protein expression. $^{29}$ Information regarding mucinous histology, histological grade, clinicopathological staging, and tumour infiltrating lymphocytes was available on a subset of cancers which had been examined for these features in previous reports. ${ }^{162} 30$

Fresh frozen polyp samples were selected to enrich the cohort for "serrated pathway" lesions as a recent report had shown that BRAF activating mutations were highly associated with these polyp types. ${ }^{34}$ Half of each bisected polyp was placed in liquid nitrogen after resection for subsequent DNA analysis while the remaining half was submitted for histological diagnosis. Eighty five polyps were classified: 43 $\mathrm{HP}$, nine MP, five SA and $28 \mathrm{AD}$ (tubular or tubulovillous adenoma) using WHO criteria. ${ }^{10} 35$ Of the 57 serrated polyps, 25 (15 HP, eight MP, and two SA) were derived from four patients with hyperplastic polyposis ${ }^{36}$ and the remaining 32 were obtained from 14 patients undergoing surgery for CRC. ${ }^{37}$ Twenty eight $\mathrm{AD}$ were derived from 11 patients undergoing surgery for CRC.

\section{Histological evaluation}

Sections $(4 \mu \mathrm{m})$ were cut from formalin fixed paraffin embedded blocks and stained with haematoxylin and eosin. Polyp cases were reviewed by two independent observers (JRJ and TH). The purpose of this review was to identify HP showing atypical histological features that have been associated with the recently documented HP variant described as "sessile serrated adenoma" (SSA). ${ }^{11-13}$ Features that were assessed included: (1) exaggerated serration, papillarity, or villosity, including serration in the lower crypt; (2) crypt irregularity, including pronounced budding, branching, or horizontally arranged crypts; (3) crypt dilatation; (4) increased crypt epithelium to stroma ratio; (5) mitoses in the upper half of crypts; (6) vesicular nuclei with prominent nucleoli in the upper half of crypts; and (7) increased production of intracellular and/or luminal mucin. ${ }^{11-13}$ HP were defined as "atypical" if four or more of these features were present. Rare discrepant findings between the two observers were resolved by consensus. HP were also partitioned for size into: (a) lesions $5 \mathrm{~mm}$ or less in size and (b) those greater than $5 \mathrm{~mm}$. The site of the polyps in the colon was also noted.

HNPCC, hereditary non-polyposis colorectal cancer; MSI-H, high level microsatellite instability; CIMP, CpG island methylator phenotype; CRC, colorectal cancer.

${ }^{*}$ CIMP status was determined using four markers (MINT 1, MINT 2, MINT 12 and MINT 31); CIMP-high, 3-4 markers methylated; CIMP-low, 1-2 markers methylated; CIMP negative, no marker methylated. tWe used Pearson's $\chi^{2}$ test or Fisher's exact test (extended) where appropriate to compare all variables. 
Table 2 BRAF and K-ras mutations in colorectal polyps

\begin{tabular}{|c|c|c|c|c|c|}
\hline Polyp type & No of cases & $\begin{array}{l}\text { BRAF } \\
\text { (V599E) }\end{array}$ & p Value ${ }^{* *}$ & K-ras & p Value ${ }^{* *}$ \\
\hline Histology & & & $<0.0001 \dagger \dagger$ & & $0.02+\dagger$ \\
\hline $\mathrm{HP}$ & 43 & $17(40 \%)$ & $<0.001 \ddagger \ddagger$ & $8(19 \%)$ & $0.02 \ddagger \ddagger$ \\
\hline Classical HP & 27 & $5(19 \%)$ & & $8(30 \%)$ & \\
\hline Variant HP† & 16 & $12(75 \%)$ & & $0(0 \%)$ & \\
\hline$M P$ & 9 & $8(89 \%)$ & & $0(0 \%)$ & \\
\hline Without atypical histology† & 1 & $0(0 \%)$ & & $0(0 \%)$ & \\
\hline With atypical histologyt & 8 & $8(100 \%)$ & $<0.0001 \S \S$ & $0(0 \%)$ & $<0.01 \S \S$ \\
\hline SA & 5 & $1(20 \%)$ & & $1(20 \%)$ & \\
\hline$A D$ & 28 & $0(0 \%)$ & $<0.0001 \%$ & $11(39 \%)$ & $0.03 \pi$ \\
\hline Characteristics of serrated polyps & 57 & & & & \\
\hline Location & & & 0.01 & & 0.07 \\
\hline Right sided & 31 & $19(61 \%)$ & & $2(6 \%)$ & \\
\hline Left sided & 26 & $7(27 \%)$ & & $7(27 \%)$ & \\
\hline Size§ & & & 0.02 & & 0.25 \\
\hline $1-5 \mathrm{~mm}$ & 38 & $13(34 \%)$ & & $8(21 \%)$ & \\
\hline $6 \mathrm{~mm}$ or more & 17 & $12(71 \%)$ & & $1(6 \%)$ & \\
\hline Unknown & 2ब & 1 & & 0 & \\
\hline Origin & & & $<0.0001$ & & 0.06 \\
\hline Hyperplastic polyposis & 25 & $22(88 \%)$ & & $1(4 \%)$ & \\
\hline Sporadic & 32 & $4(13 \%)$ & & $8(25 \%)$ & \\
\hline CIMP-status* & & & 0.002 & & 0.009 \\
\hline CIMP-high & 25 & $18(72 \%)$ & & $1(4 \%)$ & \\
\hline CIMP-low & 23 & $7(30 \%)$ & & $4(17 \%)$ & \\
\hline CIMP-negative & 8 & 1 (13\%) & & $4(50 \%)$ & \\
\hline Unknown & 1 & 0 & & 0 & \\
\hline
\end{tabular}

HP, hyperplastic polyp; MP, mixed polyp; SA, serrated adenoma; $A D$, tubular adenoma and tubulovillous adenoma; CIMP, CpG island methylator phenotype.

*CIMP status was determined using four markers (MINT 1, MINT 2, MINT 12, and MINT 31); CIMP-high, 3-4 markers methylated; CIMP-low, 1-2 markers methylated; CIMP-negative, no marker methylated.

†HP or MP showing atypical features, as described by Torlakovic et al and Goldstein et al. ${ }^{11}{ }^{13}$

¥Serrated polyps (polyps with serrated architecture) include HP, MP, and SA.

§Range 1-16 mm; mean (SEM) 5.4 (0.5) mm.

Two SA were not available for size measurement.

**We used either Pearson's $\chi_{2}^{2}$ test or Fisher's exact test where appropriate to compare all variables.

t†p value was analysed by $\chi^{2}$ test for five categories (classical HP, variant HP, MP, SA, and AD).

¥†p value is for comparison of 27 classical HP and 16 variant HP, as described by Torlakovic et al and Goldstein et al. ${ }^{11} 13$

$\S \S p$ value is for comparison of 24 polyps (16 HP and eight MP) showing atypical features and 28 polyps ( $27 \mathrm{HP}$ and one MP) lacking atypical features.

- $p$ value is for comparison of 57 serrated polyps and 28 conventional adenomas.

\section{Mutation analysis}

Genomic DNA was extracted using standard methods and amplified using previously described polymerase chain reaction primers. ${ }^{25} 38$ Samples were screened for mutations

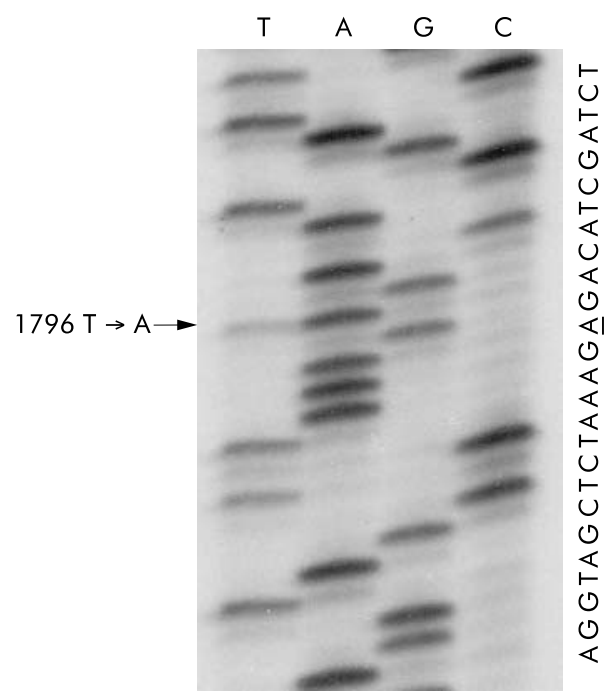

Figure 1 Representative sequence showing the BRAF V599E (T1796A) mutation. in BRAF (exon 15) and K-ras (codons 12 and 13) by restriction fragment length polymorphism (RFLP) ${ }^{32}$ 38-40 and in BRAF (exons 11 and 15) by denaturing high performance liquid chromatography (dHPLC). ${ }^{25} 41$ All products showing positive results from RFLP or dHPLC analysis were purified and sequenced manually using AmpliCycle Sequencing Kit (Applied Biosystems, Branchburg, New Jersey, USA) or automatically using Big Dye Terminator v3.1 Cycle Sequencing Kit (Applied Biosystems, Foster City, California, USA) and analysed on a ABI 3100 Genetic Analyzer.

\section{Methylation analysis}

Bisulfite modification of genomic DNA was performed as previously described. ${ }^{16}{ }^{17}$ The COBRA method was used to examine the methylation status of the four $\mathrm{CpG}$ islands (MINT1, MINT2, MINT12, and MINT31) used to determine CIMP status. ${ }^{16} 1737$ Cancers and serrated polyps were classified as CIMP-high (3-4 markers methylated), CIMP-low (1-2 markers methylated), and CIMP-negative (no marker methylated). ${ }^{16}{ }^{37}$ hMLHl promoter methylation data were obtained from previous studies. ${ }^{162937}$

\section{Statistical analysis}

Differences in frequency were assessed by Pearson's $\chi^{2}$ test, Fisher's exact test (extended), or the Student's $t$ test, and all $\mathrm{p}$ values were two sided. Multiple regression analysis was carried out using the STATISTICA 6 software package (Statsoft Corporation). A p value of $<0.05$ was considered significant. 
Table 3 Patient demographics and characteristics of colorectal cancers evaluated for $B R A F$ mutations

\begin{tabular}{|c|c|c|c|c|}
\hline Characteristic & $\begin{array}{l}\text { All patients } \\
(n=145)\end{array}$ & $\begin{array}{l}\text { Patients with } \\
\text { BRAF mutation } \\
\text { ( } \mathrm{n}=42 \text { ) }\end{array}$ & $\begin{array}{l}\text { Patients without } \\
\text { BRAF mutation } \\
\text { ( } \mathrm{n}=103 \text { ) }\end{array}$ & p Value* \\
\hline Age at operation (y) (mean (SE)) & $65.6(1.3)$ & $73.5(2.2)$ & $62.3(1.4)$ & $<0.0001$ \\
\hline Sex & & & & 0.006 \\
\hline Male & $76(52 \%)$ & $14(33 \%)$ & $62(60 \%)$ & \\
\hline Female & $69(48 \%)$ & $28(67 \%)$ & $41(40 \%)$ & \\
\hline Site relative to splenic flexure & & & & $<0.0001$ \\
\hline Right sided & $75(52 \%)$ & $35(85 \%)$ & $40(39 \%)$ & \\
\hline Left sided & $68(48 \%)$ & $6(15 \%)$ & $62(61 \%)$ & \\
\hline Unknown & 2 & 1 & 1 & \\
\hline Family history & & & & 0.002 \\
\hline Yes (HNPCC) & $18(12 \%)$ & $0(0 \%)$ & $18(17 \%)$ & \\
\hline No (sporadic) & $127(88 \%)$ & $42(100 \%)$ & $85(83 \%)$ & \\
\hline Histological grade & & & & $<0.0001$ \\
\hline Well differentiated & $2(1 \%)$ & $0(0 \%)$ & $2(2 \%)$ & \\
\hline Moderately differentiated & $87(60 \%)$ & $15(36 \%)$ & $72(70 \%)$ & \\
\hline Poorly differentiated & $51(35 \%)$ & $26(61 \%)$ & $25(24 \%)$ & \\
\hline Unknown & 5 & 1 & 4 & \\
\hline Mucinous histology & & & & $<0.0001$ \\
\hline Present & $33(33 \%)$ & $22(61 \%)$ & $11(17 \%)$ & \\
\hline Absent & $67(67 \%)$ & $14(39 \%)$ & $53(83 \%)$ & \\
\hline Tumour infiltrating lymphocytes & & & & 0.38 \\
\hline Numerous & $14(12 \%)$ & $9(26 \%)$ & $5(6 \%)$ & \\
\hline Mild infiltration & $32(28 \%)$ & $14(41 \%)$ & $20(23 \%)$ & \\
\hline No infiltration & $72(60 \%)$ & $11(33 \%)$ & $61(71 \%)$ & \\
\hline Clinicopathological stage & & & & 0.68 \\
\hline I & $22(15 \%)$ & $6(14 \%)$ & $16(16 \%)$ & \\
\hline ॥ & $72(50 \%)$ & $23(55 \%)$ & $49(48 \%)$ & \\
\hline III & $41(28 \%)$ & $11(26 \%)$ & $30(29 \%)$ & \\
\hline IV & $8(6 \%)$ & $1(2 \%)$ & $7(7 \%)$ & \\
\hline \multicolumn{5}{|c|}{$\begin{array}{l}\text { HNPCC, hereditary non-polyposis colorectal cancer. } \\
\text { *We used either Pearson's } \chi^{2} \text { test or Fisher's exact test where appropriate to compare all variables except mean } \\
\text { age at operation, for which we used an unpaired } t \text { test. } \\
\text { †Clinicopathological stage was classified according to the criteria of UICC-TNM classification. }{ }^{34}\end{array}$} \\
\hline
\end{tabular}

\section{RESULTS}

Mutually exclusive relationship between BRAF and $\mathrm{K}$-ras mutations in colorectal polyps and cancers We performed BRAF and K-ras mutation analysis on 85 colorectal polyps comprising $43 \mathrm{HP}$, nine MP, five SA, and 28 $\mathrm{AD}$, and on $145 \mathrm{CRC}$, comprising $64 \mathrm{MSI}-\mathrm{H}$ cancers (18 HNPCC and 46 sporadic) and 81 non-MSI-H cancers. In total, 26 polyps and 42 cancers containing BRAF mutations were identified in 230 samples studied (tables 1, 2). All BRAF mutations showed the same thymine to adenine transversion at nucleotide 1796, resulting in a valine to glutamine substitution at codon 599 (V599E) (fig 1). K-ras mutations were also found in 20 polyps and 43 cancers (tables 1, 2). In our study, neither polyps nor cancers simultaneously harboured both BRAF and K-ras mutations.

\section{Relationship between BRAF and K-ras mutation and MSI-status in CRC}

The oncogenic V599E mutation was identified 35 of 46 (76\%) sporadic MSI-H, seven of 81 (9\%) non-MSI-H, and in 0 of 18 $(0 \%)$ familial (HNPCC) cancers (table 1). These observations demonstrated a highly significant association between BRAF mutation and sporadic MSI-H cancers compared with nonMSI-H and HNPCC cancers $(\mathrm{p}<0.0001$ and $\mathrm{p}<0.0001$, respectively). Conversely, K-ras mutation was detected in six of $18(33 \%)$ CRC in HNPCC, 36 of $81(44 \%)$ sporadic nonMSI-H cancers, and in only one of $46(2 \%)$ sporadic MSI-H cancer (table 1). In addition, CRCs with the BRAF mutation were associated with typical features of sporadic MSI-H CRC; older age $(p<0.0001)$, female sex $(p=0.006)$, right sided anatomical location $(\mathrm{p}<0.0001)$, high tumour grade $(\mathrm{p}<0.0001)$, and mucinous histology $(\mathrm{p}<0.0001)$, compared with CRC without BRAF mutations (table 3). The clinicopathological stage of the cancer and the presence of tumour infiltrating lymphocytes showed no significant relationships with $B R A F$ mutation.

\section{Association between BRAF mutation and histopathology of polyps}

The BRAF V599E mutation was identified in 17 of $43(40 \%)$ HP, eight of nine ( $89 \%)$ MP, one of five $(20 \%)$ SA, and 0 of 28 $(0 \%) \mathrm{AD}(\mathrm{p}<0.0001)$ (table 2). Among the $43 \mathrm{HP}, 16$ showed atypical features found in SSA, as described by Torlakovic et al and Goldstein et al (fig 2). ${ }^{11}{ }^{13}$ The V599E mutation was identified in 12 of 16 (75\%) HP with atypical features (SSA) and in all of eight MP showing atypical features within the hyperplastic component. Therefore, the BRAF mutation segregated with a total of 20 of 24 (83\%) HP and MP having the atypical features used to define the HP variant termed SSA. ${ }^{11}{ }^{13}$ In contrast, the BRAF mutation occurred in only five of $28(18 \%)$ polyps ( $27 \mathrm{HP}$ and the single MP) lacking these atypical features $(p<0.0001)$. When size of lesions with serrated architecture was considered in 55 serrated polyps (43 $\mathrm{HP}$, nine MP, and three SA), the BRAF mutation was more frequently seen in polyps larger than $5 \mathrm{~mm}(\mathrm{p}<0.02)$. In addition, 12 of $15(80 \%)$ large polyps ( $6 \mathrm{~mm}$ or more) had the atypical features, contrasting with 12 of $37(32 \%)$ small polyps $(5 \mathrm{~mm}$ or less) $(\mathrm{p}<0.01)$. When location in the colon was analysed, the BRAF mutation was more highly represented in right sided serrated polyps (HP, MP, and SA) than left sided polyps $(\mathrm{p}<0.01)$. The origin of serrated polyps was also considered. In hyperplastic polyposis patients, 22 of 25 (88\%) polyps (two of two classical HP, 11 of 13 variant HP, eight of eight MP with atypical architecture, and one of two SA) harboured the BRAF mutation, while four of $32(13 \%)$ sporadic polyps (three of 25 classical HP, one of three variant $\mathrm{HP}, 0$ of one MP without serrated architecture, and 0 of three $\mathrm{SA}$ ) had the BRAF mutation $(\mathrm{p}<0.0001)$. However, multiple 


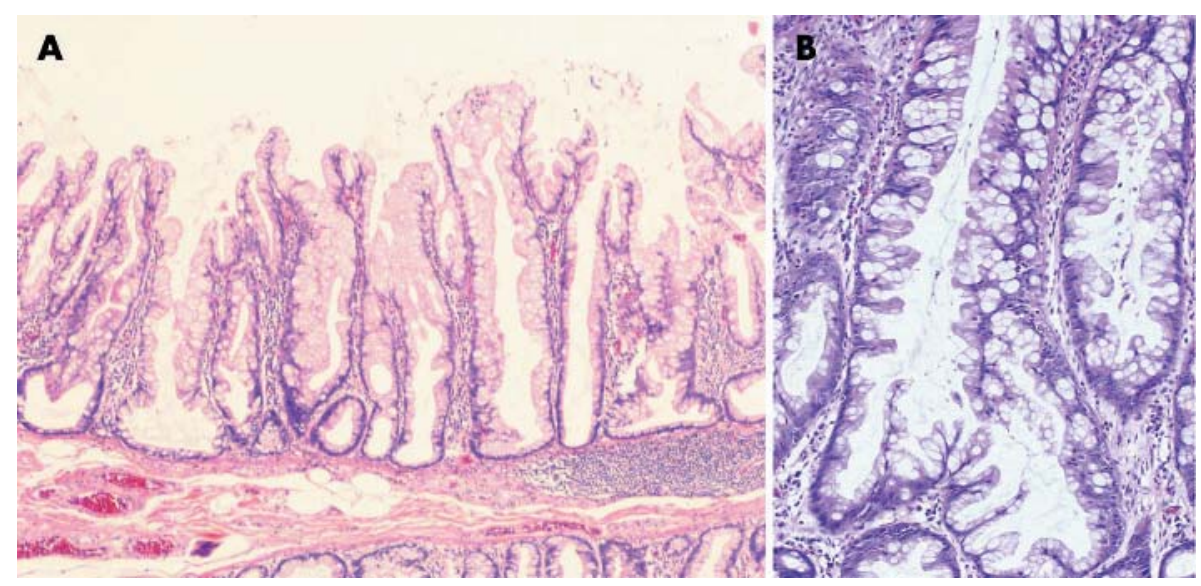

Figure 2 Haematoxylin and eosin stains of two hyperplastic polyps (HP) showing the atypical features described as sessile serrated adenoma (SSA) by Torlakovic et al and Goldstein et al. ${ }^{11}{ }^{13}$ (A) Low power view of a variant HP in which there is a hypermucinous epithelium showing crypt dilatation and horizontal extension of crypts immediately above the muscularis mucosae. (B) Medium power magnification of a variant HP showing exaggerated serration, crypt dilatation, and crypt branching, but no definite evidence of dysplasia.

regression analysis carried out on the four characteristics strongly associated with BRAF mutation (atypical histology, size, location, and origin of polyps) revealed origin within hyperplastic polyposis to be the only independent predictor of BRAF mutation $(\mathrm{p}<0.001)$.

\section{Relationship between BRAF mutation and DNA methylation in serrated polyps and CRC}

To determine if there was any relationship between $B R A F$ mutation and DNA methylation, we investigated the frequency of the BRAF (V599E) mutation in CIMP-high, CIMPlow, and CIMP-negative sporadic CRC and serrated polyps. A highly significant association was found with BRAF mutations identified in 20 of 26 CIMP-high cancers (77\%), while eight of 44 CIMP-low (18\%) and 0 of 34 CIMP-negative cancers $(0 \%)$ carried this mutation $(\mathrm{p}<0.0001)$ (table $\mathrm{l})$. Notably, when sporadic MSI-H cancers were further stratified by CIMP status, we found an even greater association. In sporadic MSI-H cases, 16 of 17 (94\%) CIMP-high cancers harboured the V599E mutation while five of nine (56\%)
CIMP-low and 0 of two (0\%) CIMP-negative cancers had the V599 mutation $(p=0.004)$ (fig 3$)$. For sporadic non-MSI-H cases, four of nine (44\%) CIMP-high cancers had the V599E mutation in contrast with three of 35 (9\%) CIMP-low and 0 of 32 CIMP-negative cancers $(\mathrm{p}=0.0008)$. When $h M L H 1$ promoter methylation was considered, it was seen in 16 of 24 cancers $(66 \%)$ with the BRAF mutation and in only four of 74 $(5 \%)$ without $(\mathrm{p}<0.0001)$. Of the four tumours with $h M L H 1$ promoter methylation but no BRAF mutation, two cases were HNPCC patient and the remaining two cases were sporadic non-CIMP-high cancers that were MSI-H. None of 18 serrated lesions tested showed $h M L H 1$ promoter methylation.

Although 22 of 25 serrated polyps obtained from subjects with hyperplastic polyposis showed BRAF mutations, there was an overall significant association between $B R A F$ mutation and DNA methylation across the 56 serrated polyps tested in the study, with 18 of 25 CIMP-high ( $72 \%$ ), seven of 23 CIMP-low (30\%), and one of eight CIMP-negative (13\%) polyps ( $p=0.002$ ) (table 2 ). Of note, four HP with the BRAF mutation were observed outside the setting of hyperplastic

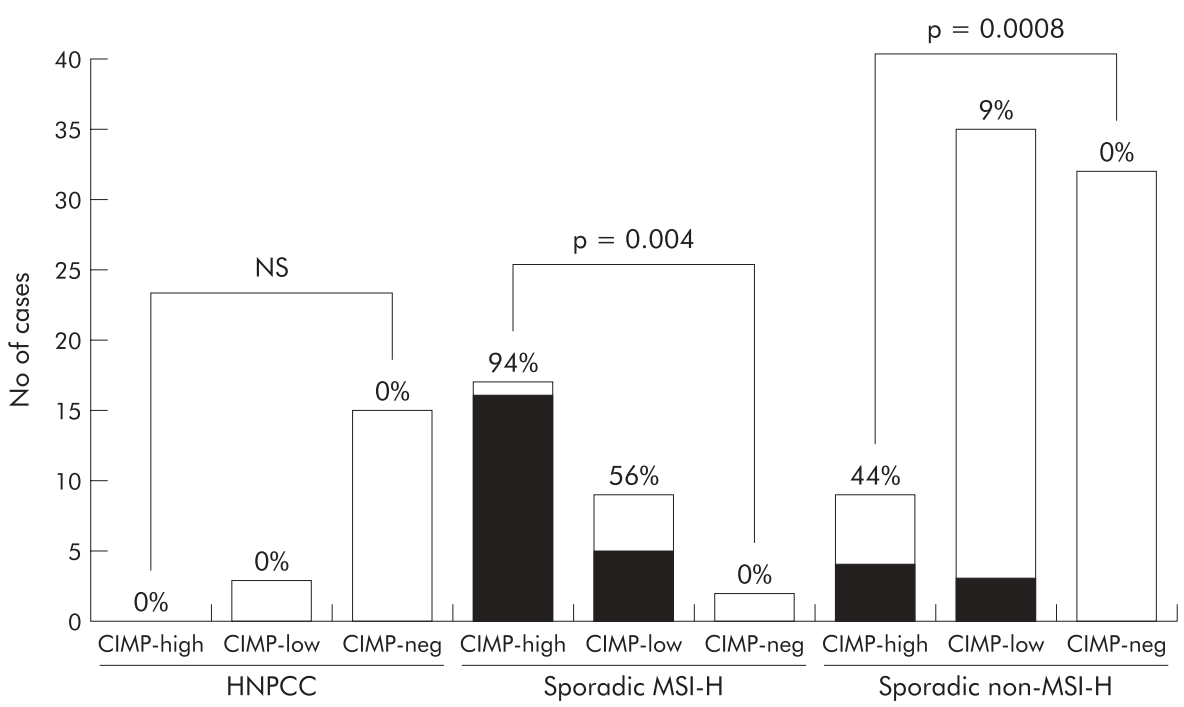

Figure 3 Histogram showing the frequency of the BRAF (V599E) mutation in hereditary non-polyposis colorectal cancer (HNPCC), sporadic high level microsatellite instability (MSI-H) cancer, and non-MSI-H cancers stratified according to CpG island methylator phenotype (CIMP) status. The percentage of $B R A F$ mutation in each group of cancers is indicated and the frequency of the mutation is represented in black. Indicated are $p$ values comparing CIMP-high, CIMP-low, and CIMP-negative cancers within sporadic MSI-H and non-MSI-H cases. 
polyposis, as defined by Burt and Jass, ${ }^{36}$ and one of these HP showed both atypical histology and CIMP-high.

\section{DISCUSSION}

In this study we showed that activating $B R A F$ mutations were strongly associated with sporadic MSI-H cancers where somatic inactivation of $h M L H I$ occurs but were not found in MSI-H cancers arising in the inherited syndrome HNPCC. Several previous studies have examined the relationship between MSI status and BRAF mutation. Yuen et al found no significant association between MSI status and BRAF mutation $^{27}$ but only a limited number of MSI-H cancers were examined. In the study of Rajagopalan et al, an association between MMR and BRAF mutation was reported ${ }^{26}$ but sporadic and inherited (HNPCC) cancers were not considered separately. Our results show clearly that inherited MSI-H cancers, which also have MMR deficiency, do not harbour $B R A F$ mutations. Rather, BRAF mutation appears to be associated with sporadic CRC, in particular those with methylation of multiple CpG islands, including in some cases the promoter of $h M L H 1$. This finding is in agreement with current reports. ${ }^{42-44}$ When CIMP status was determined using five markers (four MINTs and pl6), this trend did not change (data not shown). The BRAF mutation was also associated with the characteristics of sporadic MSI-H cancers such as female sex, mucinous histology, and advanced age at diagnosis but not with tumour infiltrating lymphocytes, a feature shared with cancers arising in HNPCC. ${ }^{29}{ }^{30}$ In contrast, hMLH1 methylation was rare in tumours without $B R A F$ mutations (four cases). In each of these cases, methylation of multiple promoters was not a concomitant feature.

The BRAF mutation was also commonly seen in CIMP-high non-MSI-H cancers (44\%) although at a lower frequency than in CIMP-high MSI-H cancers (94\%). As four of nine (44\%) of CIMP-high non-MSI-high cancers also harboured the K-ras mutation, nearly $90 \%$ of all CIMP-high cancers had either BRAF or K-ras mutations. These observations were confirmed by our findings in serrated polyps in which $h M L H I$ methylation had not occurred. ${ }^{37}$ Methylation of $h M L H 1$ is a late event that leads to dysplasia within polyps. Our data indicate that the BRAF mutation is very closely linked with DNA methylation and not specifically with $h M L H I$ methylation. These results suggest that a shared environmental factor or selection pressure lead to both DNA methylation and BRAF mutation. For example, sporadic MSI-H cancers are associated with cigarette smoking and occur predominantly in the environment of the proximal colon. ${ }^{45}$ The BRAF (V599E) mutation is frequent in tumour types prone to RAS mutation and our results agree with the concept that these are alternative ways of activating the RAS/RAF/MAP-kinase pathway. ${ }^{25-27}$ In agreement with others, we found a reciprocal association between this BRAF mutation and the K-ras mutation. ${ }^{26} 27$

The second major implication of our study is that sporadic MSI-H cancers develop from a subtype of HP and not from conventional adenomas. We found no BRAF mutation in 28 adenomas investigated. This is unlikely to be due to technical considerations such as dilution of the sample with normal DNA as all samples in this study were macroscopically dissected. Furthermore, the denaturing HPLC technique used to screen for BRAF mutations is highly sensitive across a broad range of genes. ${ }^{41}{ }^{46-48}$ In addition, others have reported the $B R A F$ mutation at a very low frequency in adenomas. ${ }^{26} 27$ Our data are similar to those of Chan and colleagues ${ }^{34}$ in that we associated BRAF mutation with colorectal polyps with epithelial serration. However, we further demonstrated that $B R A F$ mutations were associated with the variant HP described as SSA within the setting of hyperplastic polyposis. ${ }^{11}{ }^{13}$
In extraintestinal tumours, BRAF mutations also appear to occur early in tumorigenesis, for example in naevi, ${ }^{49}$ and in specific subgroups, for example serous borderline tumours which are precursors of low grade ovarian serous carcinomas. ${ }^{40}$ Activated BRAF signals through RAS/RAF/MEK/ERK/ MAP-kinase to promote proliferation but it is also known to have an antiapoptotic role. BRAF knockout mice die in utero due to increased apoptosis of differentiated endothelial cells ${ }^{24}$ and BRAF overexpression leads to protection against apoptosis by inactivating caspases after cytochrome $\mathrm{C}$ release. ${ }^{28}$ One hypothesis to explain the role of $B R A F$ in colorectal tumorigenesis would be that apoptosis evasion due to $B R A F$ activation leads to accumulation of differentiated colonocytes that characterises atypical HP and that later, when cells acquire other mutations, the effect of activated $B R A F$ is to drive proliferation.

The very high frequency (94\%) of BRAF mutations in both CRC with MSI and/or extensive DNA methylation, and in colorectal polyps with serrated morphology showing extensive DNA methylation and atypical features, strongly supports earlier work linking the development of sporadic MSI-H CRC through an alternative "serrated" pathway and suggest that BRAF mutation and DNA methylation occur as early events in this pathway. ${ }^{5222950}$ The subset of HP with atypical histological features and described as SSA are likely to be the principal precursors of MSI-H CRC. ${ }^{11-13}$ These polyps have been found with increased frequency in the colon of patients who have sporadic MSI-H CRC and also in subjects with hyperplastic polyposis. ${ }^{511} 13$ Such polyps frequently show CIMP. ${ }^{67}$ Cancers developing in patients with the condition hyperplastic polyposis in which there are large numbers of variant HP are more likely to be MSI-H. ${ }^{51}$ Methylation is related to tissue aging. ${ }^{18}$ Given the biological link between tissue aging and apoptosis and the suggestion that HP are caused by inhibition of apoptosis, ${ }^{3}$ it is conceivable that age related methylation becomes exaggerated within these polyps and serves as the basis for CIMP.

Although most of our variant HP with BRAF mutations were obtained from subjects diagnosed with hyperplastic polyposis, HP with $B R A F$ mutations, including a variant HP or SSA with both BRAF mutation and CIMP-high, were found outside the context of hyperplastic polyposis in this study, suggesting that such lesions may also arise sporadically. We would stress also that 22 of 32 of the HP occurring outside the context of hyperplastic polyposis in this study were from the distal colorectum and therefore one might not expect to find within the sporadic subset many variant HP with $B R A F$ mutations. In fact, the BRAF mutation was found in only one of 10 sporadic HP obtained from the proximal colon. This may indicate that only a fraction of proximal HP serve as precursors of CRC with BRAF mutations. In contrast, there is little evidence implicating conventional adenomas in the evolution of sporadic MSI-H CRC, ${ }^{3}$ although adenomas are the precursors to cancer in HNPCC. ${ }^{52}$ The absence of BRAF mutations in inherited MSI-H CRC, as well as in adenomas, is consistent with the suggestion that BRAF mutations do not occur as a consequence of MMR deficiency. ${ }^{43}{ }^{44}$

In this study we have shown that the BRAF mutation segregates with a variant HP described by others as sessile serrated adenoma (SSA), ${ }^{11}{ }^{13}$ at least within the setting of hyperplastic polyposis. We have also shown, in agreement with others, ${ }^{43} 44$ that the BRAF mutation segregates with sporadic MSI-H CRC showing DNA methylation. Such tumours are relatively frequent in subjects with hyperplastic polyposis, and in this syndrome we have a model for the progression of the serrated precursor lesion to CRC which is analogous to the adenoma-carcinoma model in the inherited syndrome FAP. ${ }^{53}$ Although most of our variant HP with $B R A F$ mutation were obtained from subjects diagnosed with 
hyperplastic polyposis, there were also some instances of $B R A F$ mutations in sporadic HP. Therefore, our data not only provide confirmation of the hypothesis that MSI-H CRC which arises in hyperplastic polyposis develops from variant serrated polyps, ${ }^{3}$ but also suggest that sporadic MSI-H cancers may arise from these lesions. In summary, our conclusions are based on the finding that MSI-H CRC has been linked with both hyperplastic polyposis and sporadic hyperplastic polyps, ${ }^{3}$ the fact that sessile serrated adenomas occur frequently not only in hyperplastic polyposis ${ }^{12}$ but also sporadically in the proximal colon, ${ }^{11}{ }^{13}$ and finally the fact that there are no data indicating biological differences between SSA that occur sporadically or in the context of hyperplastic polyposis.

The present study has several clinical implications. It provides a novel mechanistic basis to the clinical observation that large proximal hyperplastic-like polyps have malignant potential and may require removal when discovered during colonoscopy. Furthermore, the BRAF mutation may be employed to distinguish HNPCC from sporadic MSI-H cancers. Because it is relatively simple to detect a known single base substitution, BRAF mutations could be developed as a faecal marker of CRC. In the future, BRAF may be an attractive target for therapeutic drugs, especially as conventional adjuvant chemotherapy has recently been shown to be ineffective in the treatment of sporadic MSI-H cancer. ${ }^{54}$

\section{ACKNOWLEDGMENTS}

We would like to thank Ron Buttenshaw for expert technical assistance. This work was supported in part by the Royal Brisbane and Women's Hospital Research Foundation, the Australian National Health and Medical Research Council, the US National Institutes of Health (UO1-CA74778), and the Walter Paulson Memorial Tumor Bank. The Queensland Institute of Medical Research has approved the use of previously collected stored tissue samples, which remain anonymous to protect individuals' confidentiality.

\section{Authors' affiliations \\ T Kambara*, Conjoint Gastroenterology Laboratory, Royal Brisbane} and Women's Hospital Research Foundation and Queensland Institute of Medical Research, Brisbane 4029, Australia, and Department of Gastroenterological Surgery, Okayama University Graduate School of Medicine and Dentistry, Okayama 700-8558, Japan

L A Simms*, V L J Whitehall, K J Spring, C V A Wynter, M D Walsh, M A Barker, S Arnold, A McGivern, J Young, B A Leggett, Conjoint Gastroenterology Laboratory, Royal Brisbane and Women's Hospital Research Foundation and Queensland Institute of Medical Research, Brisbane 4029, Australia

N Matsubara, N Tanaka, Department of Gastroenterological Surgery, Okayama University Graduate School of Medicine and Dentistry, Okayama 700-8558, Japan

T Higuchi, Department of Digestive Surgery, Tokyo Medical and Dental School, Tokyo, Japan, and Department of Pathology, McGill University, Montreal, Quebec, Canada H3A 2B4

J R Jass, Department of Pathology, McGill University, Montreal, Quebec, Canada H3A 2B4

*T Kambara and L A Simms contributed equally to this work.

\section{REFERENCES}

1 Smith G, Carey FA, Beattie J, et al. Mutations in APC, Kirsten-ras, and p53alternative genetic pathways to colorectal cancer. Proc Natl Acad Sci U S A 2002;99:9433-8.

2 Kinzler KW, Vogelstein B. Lessons from hereditary colorectal cancer. Cell 1996:87:159-70.

3 Jass JR, Whitehall VL, Young J, et al. Emerging concepts in colorectal neoplasia. Gastroenterology 2002; 123:862-76.

4 Vogelstein B, Fearon ER, Hamilton SR, et al. Genetic alterations during colorectal-tumor development. N Engl J Med 1988;319:525-32.

5 Hawkins NJ, Ward RL. Sporadic colorectal cancers with microsatellite instability and their possible origin in hyperplastic polyps and serrated adenomas. J Natl Cancer Inst 2001;93:1307-13.
6 Park SJ, Rashid A, Lee JH, et al. Frequent CpG island methylation in serrated adenomas of the colorectum. Am J Pathol 2003;162:815-22.

7 Jass JR. Serrated adenoma and colorectal cancer. J Pathol 1999; 187:499-502.

8 Hawkins NJ, Bariol C, Ward RL. The serrated neoplasia pathway. Pathology 2002;34:548-55.

9 Longacre TA, Fenoglio-Preiser CM. Mixed hyperplastic adenomatous polyps/ serrated adenomas. A distinct form of colorectal neoplasia. Am J Surg Pathol 1990; 14:524-37.

10 Hamilton SR, Vogelstein B, Kudo S, et al. Carcinoma of the colon and rectum. In: Hamilton SR, Aaltonen LA, eds. Pathology and genetics: tumor of the digestive system. Lyon: IARC Press, 2000:105-19.

11 Torlakovic E, Skovlund E, Snover DC, et al. Morphologic reappraisal of serrated colorectal polyps. Am J Surg Pathol 2003;27:65-81.

12 Torlakovic E, Snover DC. Serrated adenomatous polyposis in humans. Gastroenterology 1996;1 10:748-55.

13 Goldstein NS, Bhanot P, Odish E, et al. Hyperplastic-like colon polyps that preceded microsatellite-unstable adenocarcinomas. Am J Clin Pathol 2003; 119:778-96.

14 Ahuja N, Mohan AL, Li Q, et al. Association between CpG island methylation and microsatellite instability in colorectal cancer. Cancer Res 1997:57:3370-4.

15 Hawkins N, Norrie M, Cheong K, et al. CpG island methylation in sporadic colorectal cancers and its relationship to microsatellite instability. Gastroenterology 2002;122:1376-87.

16 Whitehall VL, Wynter CV, Walsh MD, et al. Morphological and molecular heterogeneity within nonmicrosatellite instability-high colorectal cancer. Cancer Res 2002;62:6011-14

17 Toyota M, Ahuja N, Ohe-Toyota M, et al. CpG island methylator phenotype in colorectal cancer. Proc Natl Acad Sci U S A 1999:96:8681-6.

18 Toyota M, Issa JP. CpG island methylator phenotypes in aging and cancer. Semin Cancer Biol 1999;9:349-57.

19 Kane MF, Loda M, Gaida GM, et al. Methylation of the hMLH1 promoter correlates with lack of expression of hMLH1 in sporadic colon tumors and mismatch repair-defective human tumor cell lines. Cancer Res 1997;57:808-11.

20 Cunningham JM, Christensen ER, Tester DJ, et al. Hypermethylation of the $\mathrm{hMLH} 1$ promoter in colon cancer with microsatellite instability. Cancer Res 1998:58:3455-60.

21 Esteller M, Hamilton SR, Burger PC, et al. Inactivation of the DNA repair gene O6-methylguanine-DNA methyltransferase by promoter hypermethylation is a common event in primary human neoplasia. Cancer Res 1999;59:793-7.

22 Jass JR, Young J, Leggett BA. Hyperplastic polyps and DNA microsatellite unstable cancers of the colorectum. Histopathology 2000;37:295-301.

23 Peyssonnaux C, Eychene A. The Raf/MEK/ERK pathway: new concepts of activation. Biol Cell 2001;93:53-62.

24 Baccarini M. An old kinase on a new path: Raf and apoptosis. Cell Death Differ 2002;9:783-5.

25 Davies $\mathrm{H}$, Bignell GR, Cox C, et al. Mutations of the BRAF gene in human cancer. Nature 2002;417:949-54.

26 Rajagopalan $\mathrm{H}$, Bardelli A, Lengauer $\mathrm{C}$, et al. Tumorigenesis: RAF/RAS oncogenes and mismatch-repair status. Nature 2002;418:934.

27 Yuen ST, Davies H, Chan TL, et al. Similarity of the phenotypic patterns associated with BRAF and KRAS mutations in colorectal neoplasia. Cancer Res 2002;62:6451-5

28 Mercer KE, Pritchard CA. Raf proteins and cancer: B-Raf is identified as a mutational target. Biochim Biophys Acta 2003;1653:25-40.

29 Young J, Simms LA, Biden KG, et al. Features of colorectal cancers with highlevel microsatellite instability occurring in familial and sporadic settings: parallel pathways of tumorigenesis. Am J Pathol 2001;159:2107-16.

30 Jass JR, Do KA, Simms LA, et al. Morphology of sporadic colorectal cancer with DNA replication errors. Gut 1998;42:673-9.

31 Boland CR, Thibodeau SN, Hamilton SR, et al. A National Cancer Institute Workshop on Microsatellite Instability for cancer detection and familial predisposition: development of international criteria for the determination of microsatellite instability in colorectal cancer. Cancer Res 1998;58:5248-57.

32 Jass JR, Biden KG, Cummings MC, et al. Characterisation of a subtype of colorectal cancer combining features of the suppressor and mild mutator pathways. J Clin Pathol 1999;52:455-60.

33 Lindor NM, Burgart $\sqcup$, Leontovich $O$, et al. Immunohistochemistry versus microsatellite instability testing in phenotyping colorectal tumors. J Clin Oncol 2002; 20: 1043-8

34 Chan TL, Zhao W, Leung SY, et al. BRAF and KRAS mutations in colorectal hyperplastic polyps and serrated adenomas. Cancer Res 2003;63:4878-81.

35 Hamilton SR, Aaltonen LA, eds. Pathology and genetics of tumor of the digestive system. Lyon: IARC Press, 2000

36 Burt R, Jass JR. Hyperplastic polyposis. In: Hamilton SR, Aaltonen LA, eds. Pathology and genetics of tumours of the digestive system. Lyon: IARC Press, 2000:135-6.

37 Wynter CVA, Walsh MD, Higuchi T, et al. Methylation patterns define two types of hyperplastic polyp associated with colorectal cancers. Gut 2004:53:573-80.

38 Jiang W, Kahn SM, Guillem JG, et al. Rapid detection of ras oncogenes in human tumors: applications to colon, esophageal, and gastric cancer. Oncogene 1989;4:923-8.

39 Cohen $Y$, Xing M, Mambo E, et al. BRAF mutation in papillary thyroid carcinoma. J Natl Cancer Inst 2003;95:625-7.

40 Singer G, Oldt R iii, Cohen Y, et al. Mutations in BRAF and KRAS characterize the development of low-grade ovarian serous carcinoma. J Natl Cancer Inst 2003;95:484-6. 
41 Young J, Barker M, Fraser L, et al. Mutation searching in colorectal cancer studies: experience with a denaturing high-pressure liquid chromatography system for exon-by-exon scanning of tumour suppressor genes. Pathology 2002;34:529-33.

42 Deng G, Bell I, Crawley S, et al. BRAF mutation is frequently present in sporadic colorectal cancer with methylated hMLHI but not in hereditary nonpolyposis colorectal cancer. Clin Cancer Res 2004;10:191-5.

43 Koinuma K, Shitoh K, Miyakura Y, et al. Mutations of BRAF are associated with extensive $\mathrm{hMLH} 1$ promoter methylation in sporadic colorectal carcinomas. Int J Cancer 2004;108:237-42.

44 Wang L, Cunningham JM, Winters JL, et al. BRAF mutations in colon cancer are not likely attributable to defective DNA mismatch repair. Cancer Res 2003:63:5209-12.

45 Slattery ML, Curtin K, Anderson K, et al. Associations between cigarette smoking, lifestyle factors, and microsatellite instability in colon tumors. J Natl Cancer Inst 2000;92:1831-6.

46 Matyas G, De Paepe A, Halliday D, et al. Evaluation and application of denaturing HPLC for mutation detection in Marfan syndrome: Identification of 20 novel mutations and two novel polymorphisms in the FBN1 gene. Hum Mutat 2002; 19:443-56.
47 Marsh DJ, Theodosopoulos G, Howell V, et al. Rapid mutation scanning of genes associated with familial cancer syndromes using denaturing highperformance liquid chromatography. Neoplasia 2001;3:236-44.

48 Bodamer OA, Bercovich D, Schlabach M et al. Use of denaturing HPLC to provide efficient detection of mutations causing familial hypercholesterolemia. Clin Chem 2002;48:1913-18.

49 Pollock PM, Harper UL, Hansen KS, et al. High frequency of BRAF mutations in nevi. Nat Genet 2003;33:19-20

50 Jass JR. Hyperplastic-like polyps as precursors of microsatellite-unstable colorectal cancer. Am J Clin Pathol 2003;1 19:773-5.

51 Jass JR, lino H, Ruszkiewicz A, et al. Neoplastic progression occurs through mutator pathways in hyperplastic polyposis of the colorectum. Gut 2000;47:43-9.

52 lino H, Simms L, Young J, et al. DNA microsatellite instability and mismatch repair protein loss in adenomas presenting in hereditary non-polyposis colorectal cancer. Gut 2000;47:37-42.

53 Paty P, Cohen A. The adenoma carcinoma sequence in colorectal neoplasia. Gastroenterologist 1993;1:274-86.

54 Ribic CM, Sargent DJ, Moore MJ, et al. Tumor microsatellite-instability status as a predictor of benefit from fluorouracil-based adjuvant chemotherapy for colon cancer. N Engl J Med 2003;349:247-57.

\section{EDITOR'S QUIZ: GI SNAPSHOT}

\section{Answer}

From question on page 1122

Abdominal $x$ ray film showed an opaque $8 \mathrm{~cm}$ mass in the pelvis outlined by gas. The diagnosis of rectosigmoid bezoar was made. Subsequently performed barium enema confirmed the diagnosis showing an intraluminal filling defect with no attachment to the wall.

Enemas with warm saline resulted in passage of the bezoar; transanal evacuation was aided manually. Colonoscopic follow up detected no bezoar formation or other pathology.

Nearly $60 \%$ of colonic bezoars present with abdominal pain, sometimes associated with a palpable mass, abdominal distension, vomiting, constipation, or diarrhoea. The diagnosis of colonic bezoar is typically made with plain abdominal radiograph and barium enema. The method of bezoar removal depends on the site of impaction, and size, nature, and complications of the formation. Conservative management includes enemas and manual disimpaction. Colonoscopic removal is considered if enemas fail. Surgery is reserved for bezoars defeating conservative management, and for those presenting with life threatening complications such as sigmoid volvulus, haematochezia, ileus, or peritonitis.

doi: $10.1136 /$ gut.2003.24638 